International Journal of Biomedicine I June 2019 - Volume 9, Issue Suppl_1: Abstracts From the Second Russian International Conference "Cryo-electron microscopy 2019: achievements and prospects"

ORAL ABSTRACT PRESENTATIONS

SESSION TITLE: APPLICATIONS OF CRYO-EM IN MEDICINE

DOI: 10.21103/IJBM.9.Suppl_1.OR12

\title{
Abstract OR-12: Cryo-EM of Human and Parasite Proteasomes for Structure-Based Drug Design
}

\author{
Pavel Afanasyev ${ }^{1}$, Euna Yoo $^{2}$, Matthew Bogyo ${ }^{2,3}$, Paula C.A. da Fonseca ${ }^{1}$ \\ ${ }^{1}$ MRC Laboratory of Molecular Biology, Cambridge, UK \\ ${ }^{2}$ Department of Pathology and ${ }^{3}$ Department of Microbiology and Immunology, Stanford University \\ School of Medicine, Stanford, USA
}

Background: The increasing spread of Plasmodium falciparum resistance to current antimalarials, including the frontline artemisinin and its derivatives, represents a major global threat to human health and urges the development of novel medicaments. For many years, proteasomal inhibition by specific chemical compounds has been considered for use in medicine. Using cryo-electron microscopy (cryoEM), we previously demonstrated functional and structural differences between the human and the Plasmodium proteasomes that are sufficient to allow specific inhibition, particularly by the vinyl sulfone compound WLW-vs (H. Li, et al. Nature 530(7589): 233-236 2016). However, a detailed description of the molecular basis for the parasite proteasome specific targeting is still missing.

Methods and Results: Here we present a structural cryo-EM analysis of the Plasmodium 20S proteasome in the presence of the novel EY-3-123 Plasmodium proteasome inhibitor (compound 21 in E. Yoo, et al. J. Am. Chem. Soc. 140, 36, 11424-11437 (2018)). This compound has been shown to be the most potent Plasmodium specific inhibitor within a library of novel vinyl-sulfone compounds. Singleparticle analysis of this sample by extensive image classification allowed solving two high resolution proteasome structures, at about $3 \AA$, corresponding to Plasmodium and human complexes with inhibitor bound. Direct comparison of the active sites in these proteasome structures reveals the molecular basis for the Plasmodium proteasome specific inhibition.

Conclusion: The new structural information can be directly used for further development of proteasome inhibitors as potential antimalarials. This work demonstrates the high potential of cryo-EM in modern structure-based drug design.

Key Words: proteasome inhibition • cryo-EM • Plasmodium falciparum • structure-based drug design International Journal of Biomedicine. 2019;9 Suppl 1: S10-11. doi: 10.21103/IJBM.9.Suppl_1.OR12 (C)2019 International Medical Research and Development Corporation 